\title{
THE SPECTROSCOPE IN THE ELEMENTARY CHEMICAL LABORATORY.
}

By H. C. Kremers, University of Illinois, Urbana, Ill.

In many laboratories where general chemistry or qualitative analysis is being taught it is frequently the custom to have a spectroscope available for student use. The spectroseope not only teaches the student the type and relative position of the various lines of the flame spectra of some of the elements, but also serves as an aid in the identification of the alkali and alkaline earth metals. In order to have a spectroscope readily available to the students it is almost necessary that the instrument be either placed directly in the laboratory or in some convenient darkened corner. Proper supervision by the instructor also makes this condition necessary.

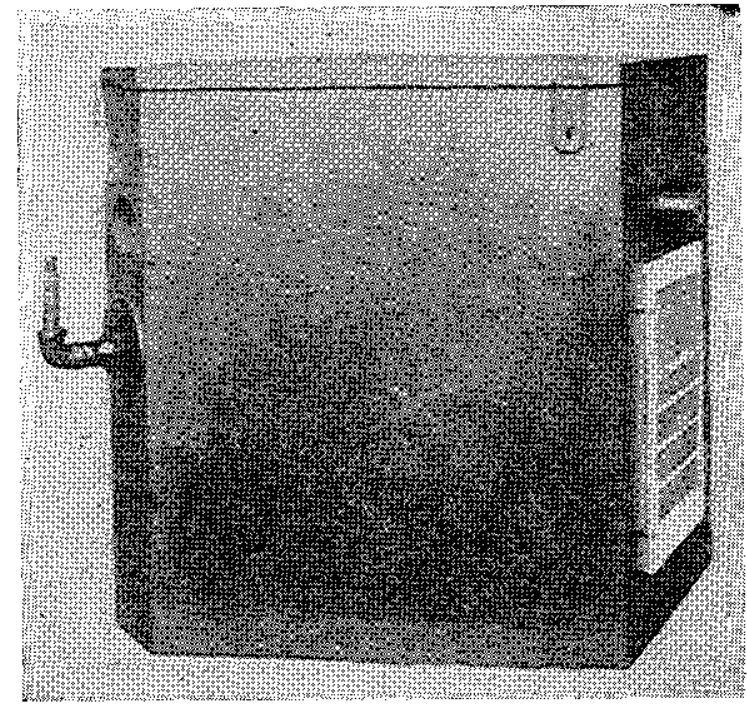

It has been the experience in this laboratory that in the hands of students the spectroscope was continually out of adjustment and being constantly exposed to laboratory gases very soon became so badly corroded as to render fine adjustment almost impossible. It was usually found necessary that the spectroscope be completely overhauled and readjusted at least every year.

Spectroscope Mounted in Boxes.

In the fall of 1917, three medium grade spectroscopes of American make were purchased to be used by our students in qualita- 
tive analysis. These spectroscopes being of a cheaper grade were rather difficult to adjust and keep in working order. In order to overcome the constant attention that these instruments required they were mounted in specially designed boxes. The accompanying photographs will bring out the idea quite clearly. These boxes were constructed of one-half inch lumber and painted a

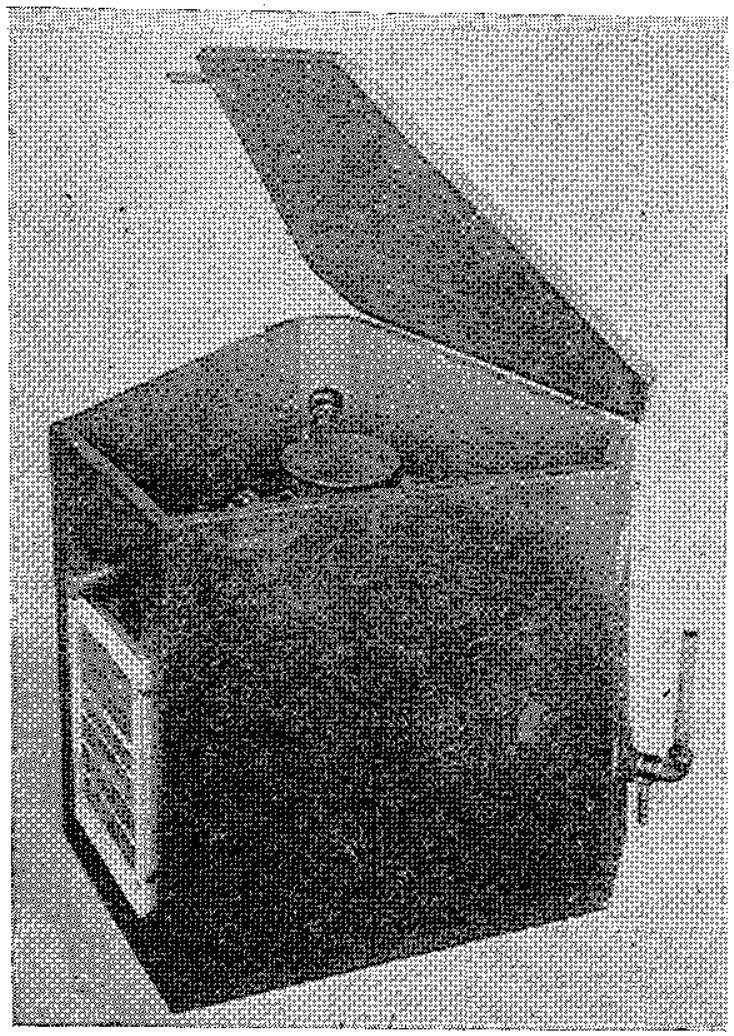

flat black both inside and out. The modified Bunsen burner in position before the slit and the fish tail illuminator for the scale are permanently mounted on the outside of the box. The light from the burners can readily gain access to the spectroscope by means of small holes cut in the box. After proper adjustment of the prism, scale and slit the box is locked and all tampering by students prevented. The large slot cut in the front of the box provides ample movement for the eye piece. The eye piece can, also, be readily focused from the outside. To facilitate the use of the instrument a chart of the flame spectra of the common elements is mounted on the box directly underneath the eye piece. 
These charts were calibrated to correspond with the calibration of the instrument.

\section{Results SATISFACTORY.}

The three spectroscopes referred to above and mounted as described have been in use continuously and at the present time are still in perfect working order. Some distinct advantages have been demonstrated.

1. The spectroseope is made readily portable and no darkened room required. It can readily be taken into the laboratory and kept under supervision and direction of the instruetor.

2. Corrosion by laboratory fumes is reduced to a minimum.

3 . Once the spectroseope is adjusted, tampering by inexperienced students is prevented.

4. With the instrument near at hand the students are more inclined to familiarize themselves with its use and advantages.

\section{THE VALUE OF FREQUENT TESTING.}

By Charles H. Sampson.

Huntington School, Boston, Mass.

Not so very long ago (the practice is still in vogue in some schools) pupils were ranked very largely on the work which they passed in for correction. This method of determining a grade for any and all abilities has changed for the better in most of our preparatory institutions. It is well that the change has taken place both for the school and for the student.

Work outside the class is often well and conscientiously done. On the other hand, there are always a few among us who seem to have been placed on earth to spoil the good intentions of the many and to grade a student because of what his home work indicates is very often an injustice in a class because a poor student and a good student receive an equal grade. Grading on the basis of grades received in frequent tests is by far the most satisfactory and most just to all concerned.

Tests should be given weekly in all subjects that are scheduled for every day. They should cover the work that has been covered during the preceding week. These examinations should be short but the questions should be selected with great care and they should aim to bring out of the mind of the student an understanding of some important principle. The making out of a test is not the matter of a few moments' time nor of the careless selection of problems or questions. A question should not be asked merely to obtain an answer but rather to determine the weaknesses that may exist in one's knowledge of any given subject. If the faults are known there can usually be a remedy. 